\title{
Non-operative Management of Posterior Shoulder Instability: An Assessment of Survival and Predictors for Conversion to Surgery at 1 to 13 Years After Diagnosis
}

Jarret Murray Woodmass, MD ${ }^{1}$, Julia Lee, MD², Nick R. Johnson, BS ${ }^{3}$, Christopher L. Camp, MD³, Diane L. Dahm, MD³, Aaron John Krych, MD ${ }^{3}$

${ }^{1}$ Boston Shoulder Institute, Boston, MA, USA, ${ }^{2}$ Sierra Pacific Orthopedics, Fresno, CA, USA, ${ }^{3}$ Mayo Clinic, Rochester, MN, USA.

Objectives: Among patients treated non-operatively for 1 year following a diagnosis of posterior shoulder instability (PSI), little is known about the the incidence of surgery between 1-13 years after injury. The purpose of this study is to define the frequency and evaluate the factors predictive for late surgical intervention of symptomatic PSI.

Methods: This study included a population-based cohort of 115 patients (14 females, 101 males) diagnosed with PSI between January 1994 and July 2012 with a minimum 5-years follow-up (mean:13.9 years; range: 5-23 years). Landmark survival analysis was performed to evaluate incidence of surgery after 1 year. Survival was estimated using Kaplan Meier method and predictors of late surgical intervention were analyzed using Cox proportional hazards regression.

Results: A total of $61 / 115$ (53\%) patients were treated non-operatively for 1 year following diagnosis of PSI. Of these, 24/61 (39\%) converted to surgery for symptomatic PSI. The overall survival free of surgery at 1 and 5 years was $53.0 \%(95 \% \mathrm{Cl} 434.7-63.0)$ and $37.1 \%(95 \% \mathrm{Cl} 29.1-47.1)$, respectively. BMI $>35$ ( $p=0.04$, HR 3.3) was predictive for late conversion to surgery. Age, gender, occupation, or history of glenohumeral dislocation were not significant. Assessing surgery as a time dependent covariate, a patient undergoing surgery was at an increased risk for radiographic progression of osteoarthritis ( $p=0.02, \mathrm{HR}=4.0,95 \% \mathrm{Cl} 1.2-13.2)$.

Conclusion: Conservative management was performed for at least one year in over half of patients diagnosed with PSI. However, long-term follow-up demonstrates that nearly $40 \%$ of these patients eventually require surgery. Increased BMI was predictive for late failure of while age, gender, history of dislocation and occupation did not show an effect. Patients who underwent surgery were at an increased risk of radiographic progression of arthritis.

The Orthopaedic Journal of Sports Medicine, 6(7)(suppl 4)

DOI: $10.1177 / 2325967118 S 00098$

CThe Author(s) 2018

This open-access article is published and distributed under the Creative Commons Attribution - NonCommercial - No Derivatives License (http://creativecommons.org/licenses/by-nc-nd/4.0/), which permits the noncommercial use, distribution, and reproduction of the article in any medium, provided the original author and source are credited. You may not alter, transform, or build upon this article without the permission of the Author(s). For article reuse guidelines, please visit SAGE's website at http://www.sagepub.com/journals-permissions. 